96 ALÉTHEIA

2015, 3(1) 96-103

Recibido: 03-06-2015

Aprobado: 08-07-2015

Palabras clave: familia, derecho de familia, investigar, investigación, tema, problema, justificación.

Keywords: family, family law, research, topic, research problem, justification

Eliana Irene Torres Adrianzen UNIFÉ etorresadrianzen@gmail.com

\section{Apuntes metodológicos para realizar un adecuado planteamiento del problema en investigaciones de derecho de familia}

\section{Methodological parameters to develop a correct research problem presentation in family law investigations}

\section{Eliana Irene Torres Adrianzen}

RESUMEN. Se analizará en el presente artículo la importancia del Derecho de Familia en el campo de la investigación jurídico social, dando a conocer los parámetros metodológicos detivados de la Metodología de la Investigación y de la experiencia adquirida, que permitan desarrollar adecuadamente el planteamiento del problema en una investigación jurídica en esta especialidad del Derecho, con la finalidad de evitar dificultades que impidan su desarrollo o se produzca su abandono.

ABSTRACT. This paper analyzes the importance of Family Law in sociolegal research, presenting the methodological parameters derived from research methodology and acquired experience which allow for an adequate development of the research problem presentation within this branch of Law, with the aim of avoiding difficulties which may impede its development or lead to its abandon. 


\section{Introducción}

$\mathbf{R}$ ealizar una investigación jurídica tomando en consideración un enfoque metodológico se convierte muchas veces en una tarea dificil para los profesionales del Derecho, quienes en su mayoría no se encuentran familiarizados con los procesos y herramientas que desarrolla la Metodología de la Investigación; lo que conlleva en el tiempo que quienes desarrollen una tesis o una investigación se sientan tan agobiados que los lleva a tomar la decisión de abandonar la tarea iniciada.

En el caso delDerecho de Familia, sibien es una rama especializada del Derecho Civil (lo cual dejaría entrever que es mucho más fácil la identificación del tema y problema de investigación), hoy en día, sus Operadores advierten que esta especialidad viene asumiendo nuevos retos en el siglo XXI, por la misma crisis que esta afrontando la Familia como núcleo fundamental de las sociedades, situación que ha generado el desarrollo de una gran problemática donde convergen aspectos de carácter jurídico, social, económico, político y hasta psicológico, los cuales determinan que sea difícil dar una solución adecuada, precisa y que cumpla con su función predictiva; $\sin$ embargo, esto debe convertirse en su fortaleza, por cuanto cobra por sí misma gran importancia y relevancia jurídica, sobre todo en el campo de la investigación jurídica.

Por ello, con el presente artículo se tratará de demostrar que realizar una investigación en esta área no constituye una tarea tan temible o dificil porque el ser humano tiene todas las condiciones necesarias, debido a que la investigación es inherente a él.

\section{INVESTIGAR E INVESTIGACIÓN}

El ser humano gracias al ejercicio de su libertad ha buscado siempre entender su entorno, comprender la razón de su existencia, aprender para crear; en otras palabras, ha estado en permanente búsqueda de datos, con la finalidad de alcanzar el conocimiento a través del contraste de sus creencias con la realidad o con aquello que le permita alcanzar la verdad valiéndose de su percepción sensorial, entendimiento y razón.
Alétheia 2015,3 (1) 97

Entonces, gracias a la búsqueda de ese conocimiento, surgen los términos investigar e investigación.

De acuerdo al Diccionario de la Real Academia de la Lengua Española (2014), la palabra "investigar" hace referencia a "bacer diligencias para descubrir algo" o "realizar actividades intelectuales y experimentales de modo sistemático con el propósito de aumentar los conocimientos sobre una determinada materia."

Ampliando el citado alcance, Cinthia Cruz del Castillo y otros (2010, p. 67 - 68), en su obra titulada "Metodología de la Investigación", refiere que investigar, citando a Mora - Ledesma y Sepúlveda (1999), se relaciona con los términos indagar, averiguar y/o buscar.

Esto conlleva a afirmar que investigar se condice con un proceso permanente y activo que ayuda a comprender en forma directa cualquier fenómeno o hecho que sea necesario analizar.

Por esa razón, cuando se trata de llevar a cabo el análisis de un determinado hecho que fluye de la realidad, se convierte realmente en una tarea dificil, por cuanto en ese proceso de averiguación o búsqueda es donde se van presentando una serie de circunstancias que están íntimamente ligadas entre sí, que hacen que se confunda o se vuelva impreciso el objeto de éstudio o unidad de análisis.

Por esa razón, y tal como lo señala Roberto Hernández Sampieri y otros (2010) en su libro "Metodología de la Investigación", los estudiantes en general crean "leyendas urbanas" o "mitos" que estigmatizan el acto de investigar como algo "complicado" y "difícil". Sin embargo, tal como se indicó en la introducción, desvirtuando la citada posición y concordando en ideas con el citado autor, el ser humano puede hacer investigación, siempre y cuando aplique los procesos y herramientas adecuadas.

Consideramos que el problema fundamental que se presenta al momento de investigar es que existe una sensación de "temor" o "miedo", hasta cierto punto aceptable, al momento en que se analiza un fenómeno o hecho derivado de la realidad; pero esto se debe, principalmente, al desconocimiento que se puede tener sobre lo que justamente se pretende analizar, sumado 
98 UNIFÉ - EPG

al hecho de no saber cómo conducir la búsqueda de información que permita aclarar el panorama de lo que será el objeto de estudio.

Por esa razón, es necesario ser constantes y disciplinados en ese proceso de búsqueda información, siendo selectivo, es decir, procurando que en la fase exploratoria de la investigación se adquiera información adecuada que permita obtener, reafirmar o ampliar conocimientos con miras a solucionar un problema.

En ese sentido, la investigación se convierte en una actividad de búsqueda que se caracteriza por ser reflexiva, sistemática y metódica, que tiene por finalidad obtener conocimientos y solucionar problemas (sean estos científicos, filosóficos, empíricos - técnicos, jurídicos - sociales), utilizando los métodos que sean más adecuados para coadyuvar a ese proceso. En otras palabras, gracias a la investigación, se ha logrado mejorar el campo de la salud con la elaboración de nuevas medicinas o procedimientos; se han innovado procesos industriales; se ha desarrollado actividades económicas incluyendo estudios que mitiguen el impacto ambiental, y, por supuesto, en el campo del derecho; se ha dado solución a conflictos socio - jurídicos a partir de la expedición, modificación e interpretación de las normas jurídicas, desarrollo de la jurisprudencia y la doctrina, fuentes que inspiran a los ordenamientos jurídicos de los países.

\section{¿POR QUÉ ES IMPORTANTE INVESTIGAR EN ÁMBITO DEL DERECHO?}

Por el ritmo de vida y sistema de trabajo muy pocas personas se ponen a pensar que día con día realizan investigación. En ese sentido, debe prescindirse de la idea que solo investiga aquel que estudia y tiene que presentar un trabajo monográfico o una tesis, o en todo caso aquella persona que está contratada por alguna institución para realizar un trabajo de investigación.

Como se ha señalado líneas artriba, la investigación es inherente al ser humano, debido a que nadie se queda conforme con lo que escucha o se le dice. Ante la ignorancia o la duda siempre procederá al proceso de indagación o búsqueda, por cuanto, concomitante a ello surge un elemento subjetivo: El interés por saber o conocer.

Investigar, como hemos visto anteriormente, permite adquirir nuevos conocimientos y resolver problemas; permite formular nuevas teorías o contradecir $y / 0$ modificar aquellas que ya existen (imvestigación básica) o conocer nuevas consecuencias prácticas (investigación aplicada).

En el caso de los profesionales del derecho, más allá de constituirse en una obligación, día con día se ven enfrentados ante la necesidad de investigar a diario sobre diferentes temáticas a partir de los diferentes dispositivos legales, sea de acuerdo a su rango o jerarquía, o por su alcance, a través de modificaciones, derogaciones o abrogaciones que se producen en el Sistema Jurídico, contrastándolos cuando fuese necesario con las posiciones sentadas por el Poder Judicial, el Tribunal Constitucional, los Órganos Colegiados en sede administrativa ${ }^{1}$, Tribunales de Solución de Controversias, así como las que se desarrollan en el Sistema Arbitral.

En otras palabras, gracias a la investigación tomando en consideración la realidad social, en especial en el campo del Derecho, se puede coadyuvar no sólo al desarrollo de la Doctrina o la Jurisprudencia, sino a realizar nuevas interpretaciones en el campo legal, que permitan y garanticen no solo la dinámica jurídica, sino su actualización y vigencia.

\section{EL DERECHO DE FAMULIA COMO OBJETO DE INVESTIGACIÓN}

Para hablar del Derecho de Familia es necesario entender a su objeto de protección: la familia.

1 Muestra de lo necesario que implica la investigación para los profesionales en Derecho, el Tribunal Constitucional, por mayoría, dejó sin efecto el precedente vinculante contenido en la STC 03741-2004-PA/ TC, conforme al cual se autorizaba a todo tribunal u órgano colegiado de la Administración Pública a inaplicar una disposición infraconstitucional cuando considere que ella vulnera manifiestamente la Constitución, sea por la forma o por el fondo.

Con esta resolución, los tribunales administrativos ya no están facultados a ejercer el denominado "control difuso de constitucionalidad". Así lo declaró el Tribunal Constitucional con motivo de resolver el Expediente $\mathrm{N}^{\circ}$ 4293-2012-PA/TC, en la demanda presentada por Consorcio Requena en contra de la Primera Sala del Tribunal de Contrataciones del Organismo Supervisor de las Contrataciones del Estado (OSCE) y el Procurador Público a cargo de sus asuntos judiciales. 
Sobre el particular, la Constitución Política del Perú de 1993, en su artúculo 402, dispone la protección de la familia y del matrimonio, reconociéndolos como institutos naturales y fundamentales de la sociedad. Es así que en concordancia con la norma fundamental, el artículo $233^{\circ}$ del Código Civil reconoce la importancia de su tegulación juridica, con la finalidad de contribuit a su consolidación y fortalecimiento.

Al respecto, el doctor Héctor Comejo Chávez (Tomol, 1985), en su obra denominada 'Derecbo Famitiar Peruano: Sociedad Convurgll', refiete que la doctrina ha señalado que en el campo sociológico y jutídico, como concepto general, la familia viene a ser el conjunto de personas unidas por los vínculos del matrimonio, el parentesco o la afinidad.

Además, tal como la misma doctrina lo reconoce, existe además del concepto de familia nuclear, otros tupos de familia ${ }^{4}$ como la extendida y la compuesta.

Sin embargo, hoy en día, hablar de una definición de familia resulta ser imposible, dado que en el siglo XXI se han experimentado una serie de cambios por las nuevas situaciones sociales que se van presentando, lo cual influye en la familia y en cada uno de sus miembros, surgiendo de esta manera una evidente crisis en la institución de la familia y por ende, surgen nuevas necesidades que demandan la atención de los Gobiemos de Turno y sus operadores.

Es decir, apatecen nuevas formas de familia: Monoparentales (cuando la mujer por lo general se queda con los hijos como consecuencia de la separación, divorcio, o quienes deciden ser madres solteras);

2 Constitución Política del Perú de 1993, Artículo 4‥ "La comunidod $y$ el Estado protegen especialmente al niño, al adolescente, a la madre $y$ al anciano en situación de abandono. También protegen a la familia y promueven el matrimonio. Reconocen a estos últimos como institutos maturales y fundamentales de la sociedad.

La forma del matrimonio y las causas de separación y de disolución son reguladas por la ley."

3 Código Civil, Artículo 233".- "La regulación jurídica de la familia tiene por finalidad contribuir a su consolidacion y fortalecimiento, en armonía con los principios y normas proclamados en la Constitución Politica del Perú."

4 El mismo doctor Héctor Cornejo Chávez (Tomo I, 1985, p. 22) y eI doctor Alex Plácido Vilcachagua (2011, p. 15), reconocen que la familia extendida, es el conjunto de personas con las cuales existe algún vínculo de parentesco o familiar; en tanto que, la familia compuesta, viene a ser el grupo social integrado por las personas que viven en una casa, bajo la autoridad det señor de ella.
Homoparentales (matrimonios entre personas del mismo sexo), las derivadas de las Uniones de Hecbo, Pareja de Hecbo, Unión Libre, Uniones Extranatrimoniales, Famitia de Hecbo, Pargia no Casada, entre otras; lo que determina una serie de situaciones derivadas o consecuencias que modifican otros institutos reconocidos en el Derecho de Familia, así como inciden en el ámbito patrimonial, labotal, de seguridad social, parentesco, alimentos, adopción, divorcio, separación, etc..

En tal sentido, y tal como lo señala el doctor Alex Plácido Vilcachagua (2011, p. 16) al comentar el artículo $233^{\circ}$ del Código Civil, el Derecho de Familia debe ser entendido como "el conjunto de normas jurídicas que regulan los vínculos familiares".

Sin embargo, si bien su tegulación proviene del Código Civil, en la actualidad (más aún dada las nuevas circunstancias de este nuevo siglo), existen una serie de normas complementarias que lo integran tanto en el ámbito familiar mismo como en otras tamas del derecho donde se puede precisar su alcance como es el caso del constitucional, penal, administrativo, comercial, tributario, notarial, laboral, seguridad social, tesponsabilidad extracontractual, entre ottas especialidades lo que determina la vigencia del Derecho de Familia y, por ende, se constituya en un área atractiva para el campo de la investigación jurídica.

\section{¿CÓMO ELEGIR EL TEMA Y PROBLEMA DE INVESTIGACIÓN EN EL DERECHO DE FAMILIA?}

Muchos autores avocados al campo de la Metodología del Derecho señalan que el primer paso pata llevar adelante una investigación reside en el problema de investigación. Sin embargo, y coincidiendo con Raúl Tafur Portilla (1995, p. 31), lo importante para iniciar una investigación es contar con un tema de estudio.

El tema de investigación, sus fuentes genetadoras y criterios.

El tema de investigación viene a ser la idea que se quiere conocer. Coincidiendo con Roberto Hernández Sampieri y ottos (2010, p. 26), existen varias 
100 UNIFÉ - EPG

fuentes que nos permiten obtener ideas para iniciar una investigación.

Adaptando dichas fuentes generadoras de ideas al ámbito del Derecho de Familia, podemos encontrar las siguientes: fuentes bibliográficas o hemerográficas especializadas que contienen comentarios en materia de derecho de familia; eventos académicos (seminarios, fórums, Diplomados, Congreso, curso de especialización donde se discutan temas actuales que se encuentran en discusión o controversia); estudios de pre y posgrado (de las cátedras de los catedráticos o debate en clase); de la revisión de la jurisprudencia o dispositivos legales del ámbito nacional e internacional en materia de familia; de la búsqueda información por internet consignando temas en discusión en el derecho de familia; de las noticias periodísticas donde se analizan casos sociales (medio escrito o televisión); de las estadísticas oficiales; del análisis de expedientes judiciales o los contenidos en los dictámenes del Ministerio Público; del intercambio de ideas de profesionales en su centro de trabajo; de un trabajo monográfico o de investigación previo, realizado por uno o de un tercero; de una experiencia personal o familiar; entre otros.

Sin embargo, y a ello se suma que una vez identificada la fuente generadora, la idea obtenida tiene que ser relevante y fáctica jurídicamente hablando, así como novedosa; pero sobre todo debe gustar y ser atractiva ${ }^{5}$ para quien investiga, tal como lo señalaba, la doctora Carmen Julia Cabello Matamala ${ }^{6}$, en sus clases magistrales de la Maestría en Derecho Civil con mención en Familia (2003). Es decir, imponer una idea o hacerlo porque lo exige el asesor de contenido o por cumplir con una formalidad no ayudará a que la investigación

5 El doctor Carlos Ramos Núñez (2005, p. 36 - 37), en su libro “Cómo hacer una Tesis de Derecho y no envejecer en el intento", agrega como pauta metodológica para selección del tema o idea de investigación que "(...) el tesista debe elegir un tema no solo que le guste sino que le colme de felicidad, que le parezca seductor y fascinante. Absténgase de buscar como tema de tesis un argumento que desagrade o que, sencillamente, no atraiga sus simpatias más profundas (...) Nunca asuma un tema que otros le sugieren mientras no advierta que a usted también le gusta de verdad (...)

6 La doctora Carmen Julia Cabello Matamala, fue Vocal integrante de la Sala Especializada en Familia de la Corte Superior de Justicia de Lima, y en la actualidad se desempeña como integrante de la Sala Civil Transitoria de la Corte Suprema de Justicia de la República, en virtud de la Resolución N 001-2015-P-PJ, de fecha 05 de enero de 2015. sea espontánea, sino una obligación con lo cual el proceso de búsqueda o de indagación se volverá tedioso y hasta aburrido y, por lo tanto, tarde o temprano, se abandonará la investigación.

En cuanto a la novedad, esta se encuentra referida a que la idea no haya sido tratada en el ordenamiento jurídico familiar peruano o habiendo sido tratada, se dé un nuevo enfoque o se actualice estudios anteriores, buscando dar una nueva orientación o alcance respecto a una investigación anterior. Lo importante como señala Roberto Hernández Sampieri y otros (2010, p. 29) es buscar la solución a los problemas.

Adicionalmente, es necesario precisar que al identificar el tema de investigación, dependiendo de su alcance, podrá responder a un tema general y, al mismo tiempo, a uno específico; no debiendo olvidar que el área temática respectiva, en el presente caso, es el Derecho Civil, y su área subtemática, el Derecho de Familia.

\section{El problema de investigación}

Cuando nos referimos al problema de investigación, lo primero que nos interesa, tras haber identificado y seleccionado el tema de investigación específico, relevante y novedoso, es conocer lo que será investigado, estableciendo cual es la duda o la falta de conocimiento existente.

Comúnmente el problema de investigación, de acuerdo a Raúl Tafur Portilla (1994, p. 81) en "Introducción a la investigación científica", está relacionado con "(...)una dificultad, una inquietud que tiene o encuentra el científico en su práctica (la investigación) acerca del becbo o conglomerado de bechos, de un aspecto de la realidad que no se resuelve directa e inmediatamente con los conocimientos existentes hasta el momento que se formula, por 10 que su solución exige implementar una actividad que se denomina investigación cientifica."

En otras palabras, el problema en sí mismo no viene a ser una pregunta ${ }^{7}$ en la investigación, sino constituye una frase, oración o proposición expresada en términos positivos, que se convierte en objeto de reflexión y sobre el cual se percibe la necesidad de conocer $y$, por tanto, de estudiar.

$7 \mathrm{El}$ problema a manera de pregunta o interrogante se relaciona con la formulación del problema. 
Para elegir el problema es necesario utilizar la "I Invia de Ideas" o Brainstorming, también denominada "Tormenta de ideas", que viene a ser una herramienta de trabajo grupal que facilita el surgimiento de nuevas ideas sobre un tema o problema determinado. Esta herramienta es usada frecuentemente en la selección del problema de investigación, el cual debe guardar relación con el tema de investigación y su respectiva área temática y, de ser el caso, su respectiva área subtemática.

En otras palabras, teniendo definido el Área Temática (Derecho Civil) y Sub Área Temática (Derecho de Familia), además del Tema de Interés (general o específico, siendo que, sobre este último, se determinará el problema), será necesario recurrir a la herramienta de trabajo antes descrita para identificar adecuadamente el problema de investigación.

En el caso del Derecho, especialmente el Derecho de Familia, interesa también observar para la precisión del problema de investigación si se trata de un Conflicto Normativo (entre normas por razón de especialidad o temporalidad; por inaplicación de las normas o interpretación inadecuada o indebida de la normativa en familia o vacíos normativos), Conflicto Jurisprudencial (contradicción en los fallos jurisdiccionales - entre las instancias del Poder Judicial competentes en el ámbito familiar) o Conflicto Doctrinal (postulación de teorías contradictorias que no resuelven la temática controvertida). Cabe señalar que estos conflictos no deben ser entendidos como situaciones aisladas, sino que estos pueden concurrir entre sí.

8 La lluvia de ideas es una técnica de grupo para generar ideas originales en un ambiente relajado.

Esta herramienta fue creada en el año 1941 por Alex F. Osborne cuando su búsqueda de ideas creativas resultó en un proceso interactivo de grupo no estructurado que generaba más y mejores ideas que las que los individuos podian producir trabajando de forma independiente; dando oportunidad de sugerir sobre un determinado asunto y aprovechando la capacidad creativa de los participantes.

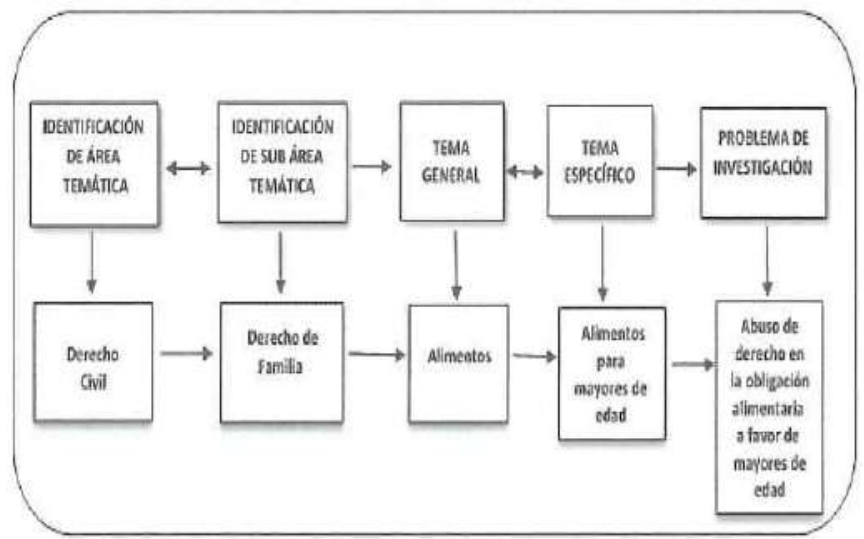

Fuente: Cuadro corresponde a claboración propia. Caso tomado del libro "Como baser una Teris de Denebo y no envejecer en el intento", del doctor Carlos Ramos Nuñez (2005).

Figura 1. Representación gráfica para la identificación y selección del tema y problema de investigación

Adicionalmente a lo anteriormente señalado, se debe precisar que es importante que el problema sea ubicado en un determinado espacio (criterio territorial) y tiempo (criterio temporal). Esto es con la finalidad de contribuir a su delimitación y se permita una investigación de carácter específico; es decir, cuanto más específico sea el problema de investigación, mejor será su desarrollo y, por ende, se podrá aplicar adecuadamente los métodos e instrumentos de comprobación.

\section{EL PLANTEAMIENTO DEL PROBLEMA}

El planteamiento de investigación es la afinación estructural de lo que viene hacer el problema de investigación. Este comprende: a) Descripción de la realidad; b) Las preguntas de investigación; c) Formulación de Objetivos; d) Justificación; e) Hipótesis.

a) Descripción de la realidad: Es conocido también como la "Enunciación del problema", y se encarga de describir las características del medio en el cual se desenvuelveelproblema.Sedescribenloshechosmás relevantes que configuran el problema; se identifican las causas (variable independiente), los efectos (variable dependiente); los sujetos involucrados en el problema (niños, niñas, adolescentes, cónyuges; 
102 UNIFÉ - EPG

entidades públicas o privadas vinculadas al ámbito familiar); ámbito donde se desarrolla el problema (entorno externo ${ }^{9}$ e interno ${ }^{10}$ ), entre otros factores.

b) Preguntas de investigación: De acuerdo a Cinthia Cruz del Castillo y otros (2010, p. 93), la pregunta de investigación forma parte del planteamiento del problema, el cual contiene a los objetivos y a la justificación de la investigación. $\mathrm{Al}$ respecto, coincidimos con esta afirmación, por cuanto las preguntas de investigación se encuentran relacionadas con la "Formulación del problema" y no con el planteamiento en sí. Estas preguntas surgen del problema a investigar, y se distinguen dos tipos:

- Pregunta general del problema, que se relaciona con la Formulación General del Problema. Esta contiene una pregunta que comunica el problema en general, es decir, el problema central de la investigación.

- Preguntas secundarias del problema, las mismas que hacen referencia a interrogantes que surgen como consecuencia de la pregunta general y sirven para desarrollar los objetivos específicos de la investigación. De alguna manera ayudan a comprender y ampliar el alcance la pregunta general del problema.

Estas preguntas van a generarse de acuerdo al enfoque del investigador $y$ las necesidades que demanda la propia investigación. Lo recomendable es realizar un listado de preguntas teniendo como referencia el problema general, las mismas que tomarán en consideración las variables identificadas en la descripción de la realidad, y de ahí, se eliminarán aquellas que son reiterativas o cuyo alcance ya se encuentre contemplada en otra preguntas.

c) Objetivos de la investigación: Roberto Hernández Sampieri y otros (2010, p. 36) al mencionar a los objetivos de la investigación, hace referencia a aquello qué pretende la investigación, es decir, de qué

$9 \mathrm{El}$ entorno externo está referido a la coyuntura y marco normativo nacional e internacional, incluyendo tratados y convenciones internacionales; políticas públicas, entre otros.

10 El entorno interno se vincula al medio de influencia directa donde se encuentran inmersos los sujetos involucrados en el problema materia de investigación. manera el estudio ayudará a resolver el problema, para lo cual se sugiere evitar posibles desviaciones en el proceso de investigación. Lo importante, señala, que los objetivos deben ser congruentes entre sí. De nuestra experiencia, estos se convierten en puntos de referencia que orientan una investigación y a cuyo logro se dirigen los esfuerzos investigativos. Consideramos que los requisitos para su formulación son los siguientes:

- Expresar adecuadamente lo que se pretende alcanzar con la investigación.

- Deben ser formulados de manera clara y precisa.

- Mantener la congruencia con la justificación del estudio y con los elementos que conforman el problema que se investiga.

- Se utilizarán verbos en infinitivo, se debe observar que los propósitos trazados sean realizables fáctica y jurídicamente, y evitar incluir criterios subjetivos.

Comprenden los Objetivos Generales y los Objetivos específicos. Los primeros guardan su correlato con la formulación general del problema; los segundos son necesarios para el desarrollo y comprensión del objetivo general.

d) La Justificación: Se encuentra relacionada con la importancia de la investigación, es decir, es necesario exponer las razones (el por qué y el para qué del estudio) de acuerdo a lo expresado por Roberto Hernández Sampieri y otros (2010, p. 39 -41). En la justificación, además, deberá destacarse la conveniencia y la relevancia jurídica y social de la investigación, pero sobre todo cuál es valor teórico y el aporte para el ordenamiento jurídico familiar (lo cual no solo incidirá en la propuesta de un proyecto normativo, sino en una serie de recomendaciones de carácter jurídico - social). Asimismo, podtá verificar la viabilidad y factibilidad del propio estudio a partir de los recursos que se encuentren disponibles.

e) La Hipótesis: Representa un elemento fundamental en el proceso de investigación. Tiene como función principal encausar la investigación que se desea llevar al efecto. Las hipótesis ocupan un lugar primordial en la investigación al proporcionar los elementos necesarios que permitirán llegar a los 
datos necesarios para resolver el problema planteado a través del o los métodos, instrumentos y técnicas de investigación acordes con el problema planteado que se pretender resolver.

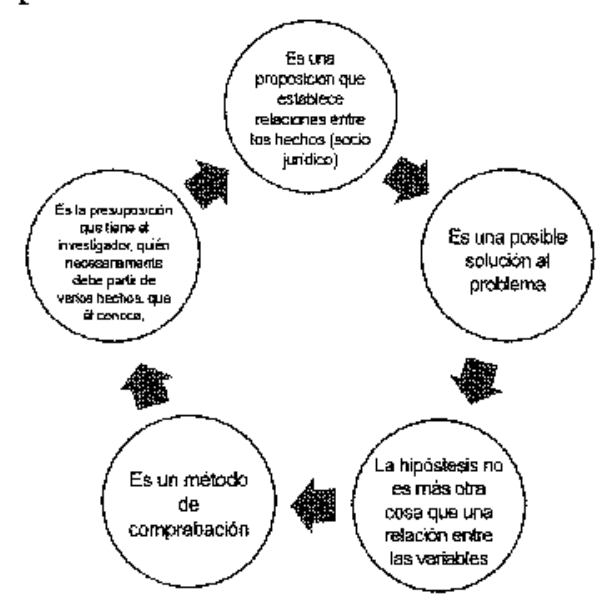

Figura 2. Acepciones - Hipótesis

Fuente propia

¿Las Hipótesis permitirán la Formulación de Hipótesis, la misma que equivale a un planteamiento que elabora el investigador a partir de la observación de una realidad que tiene explicación en una teoría.

\section{CONCLUSIONES}

1. Frente al fenómeno de la globalización, el Derecho Familiar Peruano, se encuentra afrontando nuevos retos, lo que determina que en dicho ámbito el marco normativo actual tienda a adecuarse, modificarse, derogarse o superar los vacíos legales que se presenten o tengan que concordarse con otras áreas del derecho que buscan vincularse con esta especialidad, lo cual permitirá mayor campo de acción al momento de llevar adelante una investigación de catácter jurídico social

2. Ed ser humano tiene todas las condiciones necesarias para llevar adelante ma investigación, por cuanto esta es inherente a él; por lo tanto, lo importante es conocer las hertamientas, métodos y procesos que coadyuven a su realización.

3. El tema y el problema son el punto de partida de toda investigación jurídica, lo cual demanda que
Alétheia 2015,3 (1) 103 exista obligatoriamente una actividad exploratoria donde se adquiera un conocimiento previo, que permita identificarlos en forma precisa, así como a los sujetos involuctados y sus variables extraídas de la realidad jurídico - social.

4. Cuanto más específico sea el problema de investigación, mejor será su desartollo y, por ende, se podrán aplicar adecuadamente los métodos e instrumentos de comprobación.

\section{Referencias}

Cornejo Chávez, Héctor. Derecho Familiar Peruano (1985). 5ta. Edición. Tomo I. Lima: Librería Sturdium S.A.

Cruz Del Castillo, Cinthia y otros (2010). Metodología de la Investigación. México: Grupo Editotial Patria S.A.

Gonzales Martín, Nuria. El derecho de farmilia en un mundo globalizado: Especial referencia a la adopción internacional. En: http:// www.oas.org/DIL/ESP/5\%20-\%20nuria. LR.CV.75-120.pdf.

Hernández Sampieti, Roberto y otros (2010). Metodología de la Investigación. México: McGraw-Hill / Interameticana Editores S.A.

Plácido Vilcachagua, Alex (2011). Regulación Jurídica de la Familia. En: Código Civil Comentado 3ra. edición. Tomo II. Lima: Gaceta Jutidica, pp. 15 -22 .

Ramos Núñez, Carlos (2005). Cómo hacer una tesis de Derecho y no envejecer en el intento. 3ra. Edición. Lima: Gaceta Jurídica.

Tafur Portilla Raúl (1994). Introducción a la Investigación Científica. Iima: Editorial Mantaro.

Tafur Portilla, Raúl (1995). La Tesis Universitaria. Lima: Editorial Mantaro. 